\title{
Anatomic Variation of Celiac Axis Influencing the D2 Radical Resection for stal Gastric Cancer
}

\author{
Song Bin ${ }^{1}$, Hu Haixia ${ }^{1}$, Du Juan ${ }^{2}$, Su Yanzhuo ${ }^{1, *}$ \\ ${ }^{1}$ Department of Gastrointestinal, Colorectal and Anal Surgery, China-Japan Union Hospital of Jilin \\ University, Changchun, Jilin 130033 \\ ${ }^{2}$ Internal Medicine 2, The Tumor Hospital of Jilin Changchun, Jilin 130012, P.R. China
}

Keywords: Gastric cancer, operation, celiac axis, anatomic variation

\begin{abstract}
Objective: To discuss the anatomic variation of celiac axis influencing the D2 radical operation for distal gastric cancer and its significance. Method: A retrospective analysis on the anatomic variation of celiac axis influencing operation found in 163 cases of D2 radical resection for distal gastric cancer was made. Result: 4 cases of anatomic variation of celiac axis influencing operation was found from the 163 cases, 1 case is common hepatic artery originated from left gastric artery, 1 case is left gastric artery was deficiency by 3 branch stomach arteries originating from common hepatic artery, and 2 cases are left gastric artery independently originated from aorta abdominals.

With popularization of standardization of gastric cancer operation, it is necessary to excise the routine lymph nodes in the surrounding blood vessels of celiac axis such as those beside celiac artery and left gastric artery, in front of common hepatic artery, etc. in D2 radical operation for distal gastric cancer, However, the typical type in the celiac axis accounts for 75\% [1], and the rest blood vessels all variate in different ways, leading to the risks of damaging the blood vessels and influencing the blood supply in operation. During February 2013 to February 2015, our department totally performed standard D2 radical operation for 163 cases with distal gastric cancer, and found 4 cases with coeliac trunk blood vessel variation influencing operation. Following is a summary of our experience as follows:
\end{abstract}

\section{Clinical data and method}

\subsection{General materials}

The 163 patients with distal gastric cancer who underwent standard D2 radical operation in the surgical department of stomach, intestine, colorectum and anus in China-Japan Friendship Hospital Affiliated to Jilin University during February 2013 to February 2015 were selected as research objects, to observe the variation in the branches originating from the coeliac trunk in D2 radical operation. The included cas-es all underwent gastroscopy and pathological examination after hospitalized to clari-fy the diagnosis. Wherein, there were 94 male cases, 69 female cases, whose age was 32-76 and average age was 59.8.

\subsection{Method}

The D2 lymph node excision was performed during the operation, and the sur-geon paid attention to fully exposing celiac axis, common hepatic artery, splenic artery and left gastric artery before ligating the blood vessels. After the duodenum was severed, the surgeon fully exposed gastroduodenal artery and avoid damaging it. At the 3rd, 7th, 14th day after the operation, the liver functions were reexamined, with each index being normal. The liver functions were reexamined at the 3rd, 7th, 14th day after the operation respectively. 


\section{Results}

\subsection{Variation type I}

1 Case is celiac axis gave out splenic artery and thick left gastric artery, and common hepatic artery originated from $1 \mathrm{~cm}$ from the starting point of left gastric artery. The treatment method was ligating and severing the common hepatic artery after it was given out by the left gastric artery.

\subsection{Variation type II}

1 Case is celiac axis gave out splenic artery and common hepatic artery and left gastric artery was missed. Three branch stomach arteries with the similar thickness given out by the common hepatic artery supplied blood to upper part of curvatura ventriculi minor. The treatment method was respectively ligating and severing at the root of 3 branch stomach arteries given out by common hepatic artery.

\subsection{Variation type III}

2 Case is celiac axis gave out splenic artery and common hepatic artery, and left gastric artery independently originated from abdominal aorta.

\section{Discussions}

The clinical classification and discussion about variation of celiac axis mostly refer to Michels typing [2]. The Michels type I (normal anatomical type) is the commonest, i.e the celiac axis gives out left gastric artery, splenic artery and common hepatic artery, the common hepatic artery then gives out gastroduodenal artery and arteria hepatica propria, the arteria hepatica propria is divided into left branch of proper hepatic artery and right branch of proper hepatic artery. But there are manifold variations in the blood vessels of coeliac trunk and perigastric blood vessels [3]. The report showed that $10.2 \%$ of 1,500 patients undergoing multislice helical CT (MDCT) blood vessel imaging had variation [4], and the practical operation found a higher proportion.

Currently, the standard D2 radical operation for distal gastric cancer, either la-parotomy or laparoscopic surgery, mostly adopts the method of going backward to back gap of pancreas on upper edge of pancreas along vagina vasorum of common hepatic artery to skeletonize left gastric artery and splenic artery, then occluding and dividing left gastric vessels, and excising No.7, 8a, 9, 11p groups of lymph nodes [5]. The key point of this operation is isolation along common hepatic artery and reaching the coeliac trunk, left gastric artery and splenic artery, based on the precondition that coeliac trunk belongs to normal anatomical type. If there are variations in the originat-ing blood vessel branch, such excision mode may damage the blood vessels and un-thoroughly excise the lymph nodes.

4 cases of the 163 cases in this group had coeliac trunk variation influencing the operation, accounting for $2.5 \%$. We found that the variations are mainly divided into three types: variation type I (the common hepatic artery originated from left gastric artery). If the left gastric artery is firstly isolated in operation, and left gastric artery is ligated and severed at its root, the common hepatic artery is equal to being ligated and severed synchronously. If the gastroduodenal artery is hurt when duodenum is being isolated, the liver will suffer from interruption of arterial blood supply. Variation type II (left gastric artery is missed), In operation, it is prone to mistake the first branch gastric artery given out by common hepatic artery as left gastric artery trunk, after it is ligated and severed, the surgeon continues to isolate towards coeliac trunk and mis-takenly thinks there are no main blood vessels in the gap, so he/she adopts electro-tome or ultrasound knife, which is prone to damage the second and the third branches, thereby leading to massive haemorrhage. Variation type III (the left gastric artery orig-inates from abdominal aorta), in operation, the surgeon should notice that the left gas-tric artery originates from a deep place, and will fail to find out the root of left gastric artery when reaching coeliac trunk, Now he/she should continue to explore backwards carefully along left gastric artery, avoid damaging coeliac trunk, and after reaching 
abdominal aorta, he/she should ligate at the root and avoid omitting the lymph nodes at the root of left gastric artery.

As the iconographical technology is advancing, as well as careful operation dur-ing operation, preoperative CTA is also a good means to display preoperative varia-tion of blood vessels. CTA can accurately display the invasion of blood vessel by perigastric arteries and their anatomical variation as well as tumor [6], which is favor-able for find out anatomical variation before operation to avoid damaging blood ves-sels and causing interruption of blood supply to visceral organs. Some foreign schol-ars also apply CTA to preoperative navigation for celioscopical gastric cancer excision [7].

Thus, when popularizing standardization and routinization of gastric cancer oper-ation process, we should not forget variability of blood vessels in coeliac trunk, espe-cially the variation of blood vessels influencing operation. The key to treat the coeliac trunk variation influencing operation is to understand the mutual fusion and migration relation of perigastric aponeuroses, fully expose anatomized plane, finely anatomize blood vessels and fully identify them before ligation. In the meanwhile, application of iconography is also a good means for preoperative judgment.

\section{Conclusions}

In the radical resection for gastric cancer, the operator should consider the possibility of coeliac trunk blood vessel variation, carefully anatomize the variating blood vessel to avoid damaging the blood supply for visceral organs.

\section{References}

[1] Saeed M, Murshid KR, Rufai AA, etal. Coexistence of multiple anomalies in the celiacmesenteric arterial system[J].Clinical anatomy, 2003,16(1): 30-36 .

[2]Li Tiegang, Yao Hongliang, Yu Dan, Hepatic Artery Variation in Radical Operation for Carcinoma of Stomach and Its Significance [J]. Chinese Journal of Modern Opera-tive Surgery, 2006,10 (6):418-419

[3]Song SY, Chung JW, Yin YH, etal. Celiac axis and common hepatic artery variations in 5002 patients: systematic analysis with spiral CT and DSA[J]. Radiology, 2010,255(1):278-288

[4] Yi Wang,Cheng Cheng,Lu Wang, etal. Anatomical variations in the origins of the celiac axis and the superior mesenteric artery: MDCT angiographic findings and their probable embryological mechanism[J]. European Radiology,2014,24(8):1777-1784

[5] Xiao Junfeng, Ji Ling, Ruan Xiaojiao, et al. Application of "Pancreas Centre Dissection Method" to Celioscope-assisted Lymph Nodes Excision in Distal D2 Radical Operation for Gastric Cancer [J]. Chinese Journal of Minimally Invasive Surgery, 2013,13(2):119-122

[6] Zhu Jin, Liu Te, Ling Rennan, et al. Application Value of Displaying Perigastric Arteries and Their Variation by CTA in Preoperative Evaluation on Gastric Cancer. Journal of Clinical Radiology, 2011,30 (8):1150-1152

[7] Fujiwara M, Kodera Y, Satake H, et al. Navigation for laparoscopic gastrectomy with 3dimensional computed tomography(3D-CT) [J]. Hepato-gastroenterology, 2008,55(85):1201-1205. 